Original article

\title{
Role of HRCT imaging in predicting the visibility of Round window (RW) on patients underwent cochlear implant surgery
}

\author{
Mozafar Sarafraz ${ }^{\mathrm{a}}$, Mahsa Heidari ${ }^{\mathrm{a}}$, Arash Bayat ${ }^{\mathrm{b}}$, Mohammad Ghasem Hanafi $^{\mathrm{a}}$, Ali Fahimi ${ }^{\mathrm{a}}$, \\ Maryam Farasat $^{\mathrm{a}}$, Nader Saki ${ }^{\mathrm{a}, *}$, Javad Molaei ${ }^{\mathrm{c}}$ \\ ${ }^{a}$ Hearing Research Center, Imam Khomeini Hospital, Ahvaz Jundishapur University of Medical Sciences, Ahvaz, Iran \\ ${ }^{\mathrm{b}}$ Musculoskletal Rehabilitation Research Center, School of Rehabilitation Sciences, Ahvaz Jundishapur University of Medical Sciences, Ahvaz, Iran \\ ${ }^{\mathrm{c}}$ Emam Khomeini hospital, Ahvaz Jundishapur University of Medical Sciences, Iran
}

\section{A R T I C L E I N F O}

\section{Keywords:}

Cochlear implantation

Round window

Computed tomography

Ahvaz

\begin{abstract}
A B S T R A C T
Introduction: High-resolution computed tomography (HRCT) magnifies the role of preoperative imaging for detailed inner and middle ear anatomical information and enhances more efforts for better dependent correlation and measurements of round window (RW).

Objectives: The aim of this study was to find an appropriate way by HRCT imaging for the prediction of the visibility of the round window during cochlear implant (CI) surgery.

Methods: The study was conducted using hospital information from patients referring the Khuzestan Cochlear Implant Center (2017-2018). 87 patients underwent cochlear implant surgery, 56 patients with high resolution CT scans were included in the study. All patients underwent surgical treatment via posterior tympanotomy approach.

Results: 56 patients were examined. Patients were divided into three groups: children (0-12 years) and adolescence (18-13 years) and adults (19-59 years), of whom 45 patients (80.4\%) were children, and 3 patients were adolescence $(5.4 \%)$ and 8 patients $(14.3 \%)$ were adults. 22 patients $(39.3 \%)$ were fully visible and 13 patients $(23.2 \%)$ were partially visible and $21(37.5 \%)$ patients were difficult to visualize. In high-resolution imaging studies, mastoid 2 cases (3.6\%) were poorly aerated and 8 cases $(14.3 \%)$ were moderately aerated and $46(86.1 \%)$ were well aerated. The amount of round window overhanging in 43 patients $(76.8 \%)$ was 4.4 and in 12 patients $(21.4 \%)$ was $3.4 \%$.

Conclusion: The presence of the air cell around the facial recess may provide easier access to the facial recess. There was a significant relationship between the RW in the operation and the angle between the RW and the facial nerve and the coronal axis. There is no significant relationship between the visibility of the RW and the facial recess, Vertical height RW and facial nerve (FN), the distance between the FN and the posterior canal in the axial, the angle between the FN and inner margin of RW, and the angle between the FN and the tympanic annulus with the inner margin RW in the Axial and FRL was not found.
\end{abstract}

\section{Introduction}

A person with deep sensorineural hearing loss is potential candidate for cochlear implant (CI) and should be evaluated in a professional hospital under the supervision of a doctor performing surgery. ${ }^{1}$ The evaluation includes: precise evaluation of hearing and behavior, imaging to determine the condition of the inner ear and auditory nerve, and evaluation of other medical conditions. Individuals with congenital biliary hearing loss who are eligible for auditory cochlear implants and are unable to adapt to hearing aids such as earphones are eligible for surgery. ${ }^{2,3}$ The primary way to enter the electrode array into the cochlea is to open the mastoid and posterior tympanic incision space through a circular valve. The Round window provide easy access to the Scala Tympani and are in result the easiest way to place the electrode organizer. Circular hinges are usually covered and cannot be seen directly. ${ }^{4}$ High-resolution CT scans provide very useful information about the inner and middle ear anatomy, as well as accurate measurements of RW. ${ }^{5}$ Posterior approach tympanotomy is the most common method of cochlear implant surgery worldwide. ${ }^{6}$ Today, the importance of maintaining hearing during cochlear implants indicates the use of a round window (RW) and its importance as a method of embedding a cochlear implanted electrode. ${ }^{7}$ The round window passage for cochlear implant

\footnotetext{
* Corresponding author.

E-mail address: ahvaz.ent@gmail.com (N. Saki).
} 
(CI) reduces the risk of intracochlear damage. Since there is no need to drill cochlea, the possibility of bone fragments entering Scala Tympani is limited. ${ }^{8}$ This path provides the proximal medial premature position of the bone spiral laminate as it passes through the electrode, which increases the likelihood of stimulating residual nerve dendrites. ${ }^{4}$

The round window is used as an accurate anatomical landmark for cochlear implants, so understanding the apparent morphology and anatomical differences before cochlear implantation is a necessary condition for successful cochlear implantation. ${ }^{2}$ The round window niche has two bony overhangs, one anteroinferiorly and the other posteriorly, called the anterior and posterior pillars respectively. ${ }^{7}$ The RW opening was found directed posterior in $15 \%$, posterior-inferior in $40 \%$, and inferior in $45 \%$. In approximately one-third of population, the RW niche is fully or partially covered by false membrane. ${ }^{3} \mathrm{~A}$ lot of research has been done, especially by Kashio. He tried to predict the round window visibility by radiological measurements that depend mainly on the external auditory canal (EAC) angle. ${ }^{8}$

The possibility of visually examining a round window by posterior tympanotomy during cochlear implant surgery is variable. Therefore, St. Thomas Hospital introduced a type of RW: Type I for observation during cochlear implant (CI), in which RWM was completely exposed. Type II, RWM has been partially exposed (IIb, IIa). Type III is unable to see RWM, in which case RWM is located near the facial nerve (FN). ${ }^{9}$ The aim of this study was to find an appropriate way by HRCT imaging for the prediction of the visibility of the round window during CI surgery.

\section{Material and methods}

The study was conducted using hospital information from patients referring the Khuzestan Cochlear Implant Center (2017-2018). 87 patients underwent cochlear implant surgery, 56 patients with high resolution CT scans were included in the study. All patients underwent surgical treatment via posterior tympanotomy approach. Individuals with inner or middle ear anomalies were excluded from the study. In addition, patients with chronic inflammatory middle ear diseases were also excluded as those diseases may affect the visibility of RW during surgery.

All the axial HRCT images of the temporal bone were obtained parallel to the supra-orbito-meatal line baseline, SOMATOM definition AS + 16-slice (Siemens). slice thickness of $0.75 \mathrm{~mm} .120 \mathrm{kV}$, care dose UL $200 \mathrm{~mA}$ with a matrix size of $512 * 512$ was used.

\subsection{Radiologic measurement}

Ct scan is analyzed for: Fig. 1 Mastoid, which are divided into three types of well, moderate and poor aerated.

Fig. 2 Position of the sigmoid sinus in axial: First, straight line is

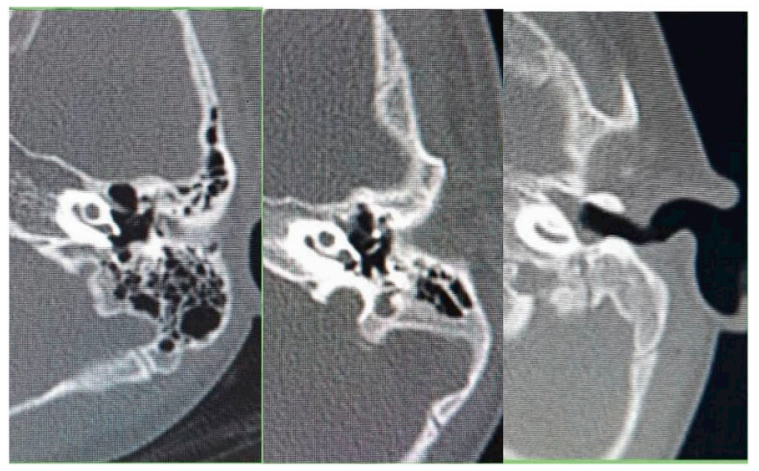

Fig. 1. Representative axial images of pre-operative high-resolution temporal bone CT scan showing mastoid that is (a) well aerated, (b) moderately aerated, and (c) poorly aerated.

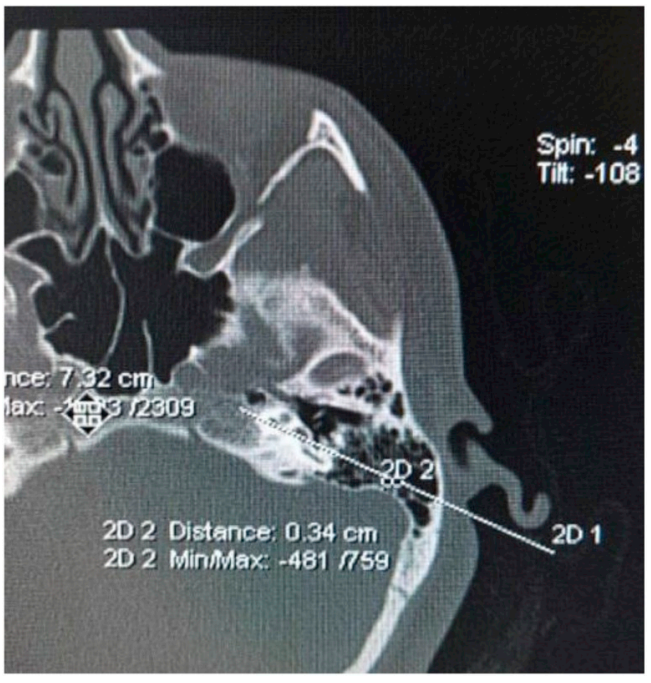

Fig. 2. Representative axial image of pre-operative high-resolution temporal bone CT scan illustrating the distance measured from the line drawn through round window and facial nerve to the anterior aspect of the sigmoid sinus.

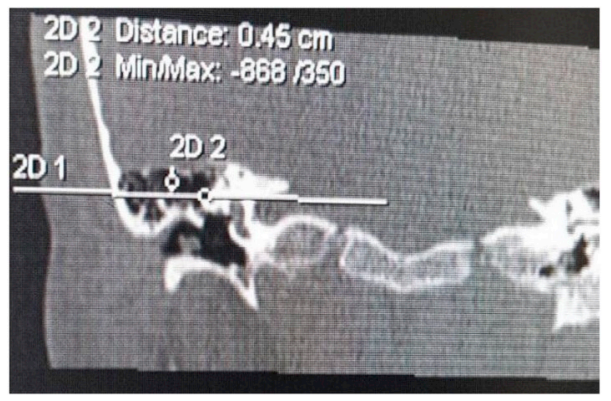

Fig. 3. Representative coronal image of pre-operative high-resolution temporal bone CT scan illustrating the distance measured from the line drawn through horizontal semicircular canal to the tegmen.

drawn from the middle of the RW and nerve 7, and then it is drawn from the perpendicular line of the axis to the front of the sigmoid sinus, and finally it is reported in millimeters.

Fig. 3 Height of the tegmen: to evaluate the height of tegmen, straight line passing through the horizontal SCC axis in the subsequently drawn coronal image, subsequently, the perpendicular line from this axis extends to the lower part of the tegmen, and the measured value is reported in millimeters.

Fig. 4 Air cell around the facial recess: An axial image of the presence or absence of air cells around the facial recess is used.

Fig. 5 Overhanging bone around round window: 4 consecutive axial cut starting from the highest point of the RW membrane and continuously downward. The number of counts representing the full thickness bony overhanging of the round window, 4 is calculated. Therefore, if the two cut have a full thickness bony overhanging, it is calculated to be 2.4 or 0.5 . This variable is compared to the amount of round window visibility during surgery.

Fig. 6 The angel between the external ear canal and the basal turn Cochlea: EAC line was drawn between the bony-cartilaginous junction of the posterior wall of the EAC and the tympanic annulus. Basal turn line was drawn through the center of the basal turn. EAC angle is the angle between both lines (EAC and basal turn lines).

The facial nerve width: is the vertical distance between the anterolateral part of the FN and the EAC line passing through the tympanic annulus. $^{8}$

Facial nerve location to the basal turn of the cochlea: the vertical distance between the anterolateral part of the FN and the basal turn 


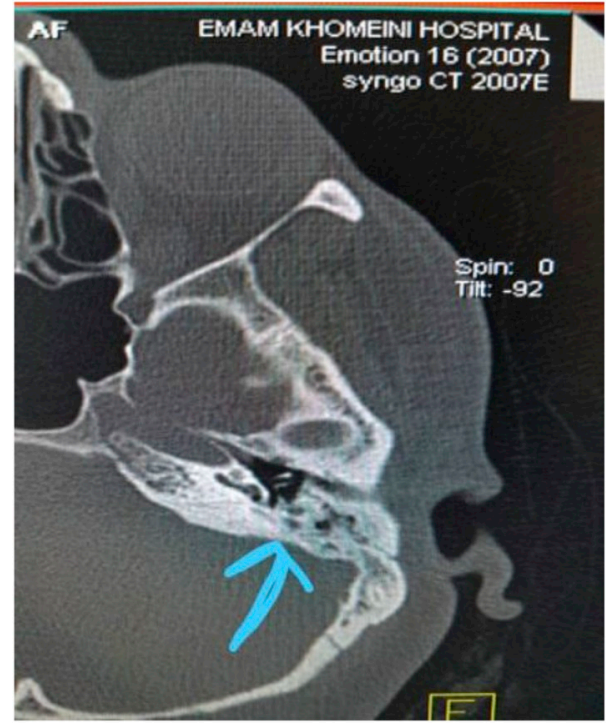

Fig. 4. Representative axial image of pre-operative high-resolution temporal bone CT scan illustrating an air cell (arrow) anterior to facial nerve.

line. ${ }^{9}$ The angle between the round window, the facial nerve and the coronal axis.

The vertical distance between the round window and the facial nerve. The position of the facial nerve relative to the Cochlea axis: upper anterior, lower posterior.

facial-round window line on axial:

Type 0: posterior to the RW.

Type 1: between the RW.

Type 2: anterior to the RW.

\subsection{Surgical classification}

Surgeons and radiologists reviewed and judged the results of HRCT. Surgeons blinded to the HRCT image results of the patients. The visibility of RW niche was judged and graded after finishing the posterior tympanotomy before drilling the cochlea. Before judging all forward and lateral bones to the FN should be drilled and the EAC should be completely thinned. The round window will be divided into three types: I, II and III. Visibility of Round window (A: invisible or almost visible, B: partially visible, C: fully visible)

\subsection{Statistical analysis}

SPSS version 22 will be used in order to compare results. The

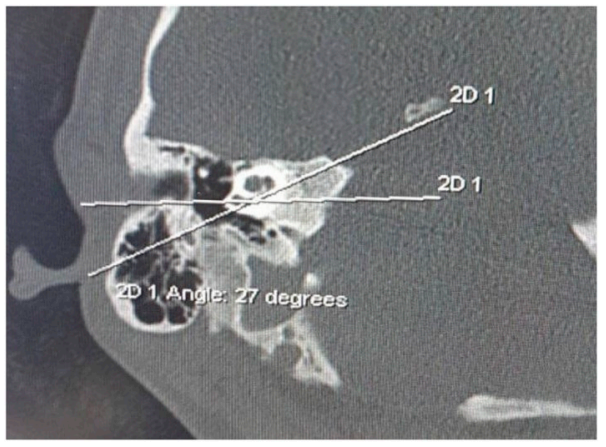

Fig. 6. Measurement of the angle between RWM, FN and the coronal axis.

Pearson correlation coefficient and R2 is used to test the relationship between the results. When the $\mathrm{p}$ value is less than 0.05 , the statistical difference will be significant.

\section{Results}

In this study, 56 patients were examined. Patients were divided into three groups: children ( $0-12$ years) and adolescence (18-13 years) and adults (19-59 years), of whom 45 patients (80.4\%) were children, and 3 patients were adolescence (5.4\% puberty) and 8 patients (14.3\%) were adults. Men accounted for $37.5 \%$, and women accounted for $35 \%$, and their combined and calculated percentage is $62.5 \%$. For 36 patients of the whole group round windows approach was used, while for 20 patient's cochleostomy approach was used. (Table 1).

The visibility round window was classified as above during surgery: 22 patients (39.3\%) were fully visible and 13 patients $(23.2 \%)$ were partially visible and $21(37.5 \%)$ patients were difficult to visualize. In high-resolution imaging studies, mastoid 2 cases $(3.6 \%)$ were poorly aerated and 8 cases $(14.3 \%)$ were moderately aerated and $46(86.1 \%)$ were well aerated. In this study, 17 children were fully visible, 11 were partially visible, and 17 were difficult to visible. In the adult group, 4 cases were fully visible 1 , partially visible and 3 cases were difficult to visible. The amount of round window overhanging in 43 patients was $76.8 \%, 4.4 \%$ and in 12 patients, $21.4 \%$, and 3.4. Aircell in facial recess was found in 37 cases of $66.1 \%$. The angle between RW and FN and the coronal axis was minimum: $20^{\circ}$ and maximum: $50^{\circ}$ with mean: 36.3 and SD: 7.73. $(P=0.04)$ In all cases, the facial nerve position was relative to the inferoposterior cochlea axis.

In the study, 8 FRL types were typed 0,22 were typed 1 and 8 were typed 2, which was correlated with RW in chi-square test with $\mathrm{p}=0.54$.
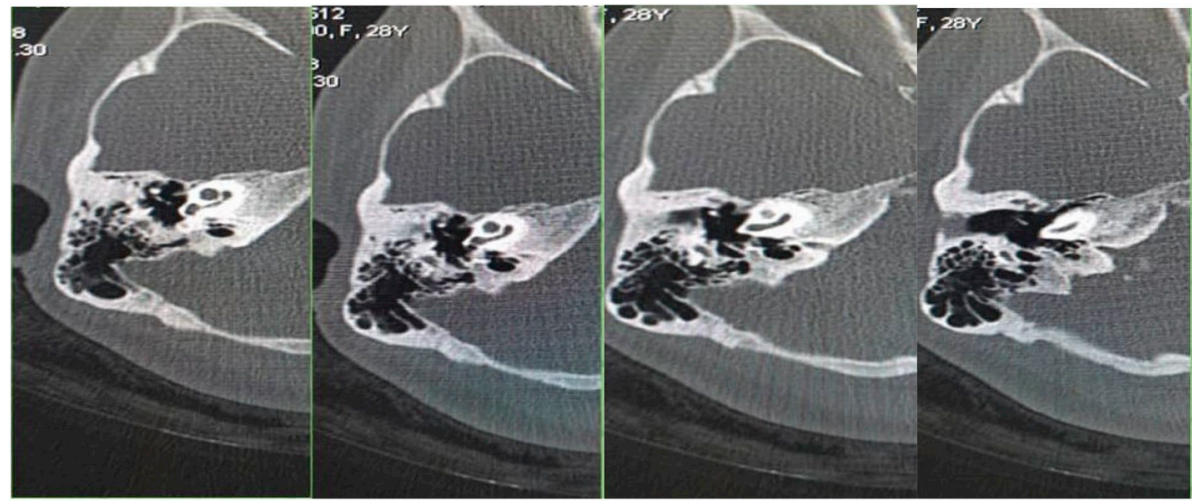

Fig. 5. Representative axial images of pre-operative high-resolution temporal bone CT scan illustrating bony overhang around round window in four consecutive slices from superior to inferior. 
Table 1

Descriptive; FWD, FRA, FRAA, EAC/CBT, WFR, FN/CBT, RW/FN/CA, VRW/FN, according to visibility of RW.

\begin{tabular}{|c|c|c|c|c|c|}
\hline & Mean & Std. Deviation & Minimum & Maximum & Sig. \\
\hline distance from facial nerve to posterior canal wall in axial view & 0.4714 & 0.07331 & 0.30 & 0.63 & 0.761 \\
\hline the angle between facial nerve and inner margin of round window in axial view & 40.0179 & 8.30004 & 25.00 & 56.00 & 0.366 \\
\hline the angle between facial nerve and tympanic annulus to inner margin of round window in axial view & 43.2308 & 8.36383 & 29.00 & 67.00 & 0.188 \\
\hline external auditory canal relative to cochlear basal turn & 18.1964 & 7.89343 & 6.00 & 38.00 & 0.141 \\
\hline width of facial recess & 0.6163 & 0.16776 & 0.30 & 1.09 & 0.371 \\
\hline location of facial nerve to cochlear basal turn & 0.2180 & 0.06062 & 0.08 & 0.35 & 0.703 \\
\hline angle between round window, facial nerve and coronal axis & 36.3571 & 7.73758 & 20.00 & 50 & 0.040 \\
\hline vertical distance between round window and facial nerve & 0.4570 & 0.08349 & 0.30 & 0.75 & 0.257 \\
\hline sigmoid sinus & 0.6630 & 0.23450 & 0.29 & 1.50 & 0.327 \\
\hline height of tegmen & 0.9515 & 0.24068 & 0.44 & 1.47 & 0.520 \\
\hline
\end{tabular}

\section{Discussion}

The aim of this study was to find an appropriate way by HRCT imaging for the prediction of the visibility of the round window during CI surgery. At the moment, surgeons routinely observe preoperative CT scans and examine the review mental list to ensure that the inserted cochlear implant has no anatomical obstruction.

If there are any anatomical variations, the surgeon will not only be cautious, but will also better discuss alternatives and their potential risks with patients or relatives. There are many variables about CT scan seen by the surgeon before surgery. Our goal is to standardize various radiological variables to predict the challenges surgeons may face during surgery.

Our results showed that the reduction in mastoid bone aeration is associated with the difficulty of mastoidectomy $(\mathrm{p}=0.002)$. According to this study, the lack of air around the facial recess was associated with more cortical mastoidectomy $(\mathrm{p}=0.002)$, and the results were matched with Edward Park and colleagues studies in 2015. In other words, the presence of an air cells around the facial recess can provide easier access to the facial recess. The round window overhang cannot predict the severity of the connection to the round window method ( $p=0.8)$, and the results of the study Park \& Associates in 2015 confirmed our results. ${ }^{10}$

The extent of round window overhanging obtained in this study was significant $(P=0.04)$, which is consistent with the results of Edward Park et al. (2015) $(\mathrm{p}=0.02)$. Exposure to round window, the amount of overhanging bone thickness can cause problems for the surgeon. The overhanging thick bony can stop the precise position and orientation of the RW. This overhanging bone thickness must be drilled, so that we can easily insert the electrode into the pre-lymphatic cochlea. The thickness of the overhanging bone is not associated with more difficulty in reaching the RW.

It can be seen that there is no significant correlation between the visibility of round window and the width of face recess $(P=0.37)$, the results were similar to the study of Fouad et al., in 2017 $(\mathrm{P}=0.941363)$. There is no significant relationship between the height of vertical RW and FN ( $p=0.25)$, while in Fouad et al. study it was statistically significant $(p=0.006552) .{ }^{11}$ In the current study, there were no significant relationship between the visibility of RW and distance between $\mathrm{FN}$ and posterior canal in axial $(\mathrm{P}=0.6)$, While in $\mathrm{Su}$ et al. Studies its significant $(P=0.00)$. There is no relation between the angle of facial nerve and tympanic annulus to inner margin of round window in axial view $(\mathrm{P}=0.18)$, whereas $\mathrm{Su}$ et al. reported a significant relationship between them $(P=0.00) .{ }^{12}$ In our study, we didn't see any relation between visibility of RW and types of FRL, unlike the result of Su et al. that they reported the FRL type 0 causes Full visibility of RW during surgery.

\subsection{Limitations}

The main limitation of the current study appears to be a relatively small sample size. low number of patients may not be statistically significant. Judgment seems to be more precise studies are needed in this area with a high volume of patients.

\section{Conclusion}

The results of this study showed that mastoid aeration may be effective in predicting the degree of difficulty in mastoidectomy. The presence of the air cell around the facial recess may provide easier access to the facial recess. There is a significant relationship between the degree the degree of RW visibility and the angle between the RW, the facial nerve and the coronal axis during surgery but there is no significant relationship between the visibility of the RW and the facial recess, Vertical height RW and FN, the distance between the FN and the posterior canal in the axial, The angle between the FN and inner margin of RW, and the angle between the FN and the tympanic annulus with the inner margin RW in the Axial and FRL was not found.

\section{Informed consent}

Informed consent was signed prior to participation in the study.

\section{Funding/Support}

Ahvaz Jundishapur University of Medical Sciences financially supported this study.

\section{Declaration of competing interest}

The authors report no conflicts of interest. The authors alone are responsible for the content and writing of the paper.

\section{Acknowledgements}

This article was the result of the dissertation thesis on HRC-9708 issued by the Committee on Ethics of the University (approved number IR.AJUMS.REC.1397.494). This study was funded by the ViceChancellor for Research at the University. We thank all our colleagues in Hearing research center, Ahvaz, Iran.

\section{References}

1. Adunka O, Unkelbach MH, Mack M, Hambek M, Gstoettner W, Kiefer J. Cochlear implantation via the round window membrane minimizes trauma to cochlear structures: a histologically controlled insertion study. Acta Otolaryngol. 2004:124:807-812.

2. Aslan A, Tekdemir I, Gunhan K, Eskiizmir G, Elhan A. Anatomic observations on variations of the round window niche and its relationship to the tympanic membrane. Mediterr. J. Otol. 2006;2:52-57.

3. Saki N, Bayat A, Hoseinabadi R, Nikakhlagh S, Karimi M, Dashti R. Universal newborn hearing screening in southwestern Iran. Int J Pediatr Otorhinolaryngol. 2017;97:89-92.

4. Briggs RJ, Tykocinski M, Stidham K, Roberson JB. Cochleostomy site: implications for electrode placement and hearing preservation. Acta Otolaryngol. 
2005;125:870-876

5. Cohen D, Blinder G, Perez R, Raveh D. Standardized computed tomographic imaging and dimensions of the round-window niche. Int Tinnitus J. 2005;11:158.

6. El-Anwar MW, ElAassar AS, Foad YA. Non-mastoidectomy cochlear implant approaches: a literature review. Int Arch Otorhinolaryngol. 2016;20:180-184.

7. Hanafi MG, Saki N, Bahmani S. Investigating the effect of cochlear size in insertion of electrode depth in patients with cochlear implantation evaluated by CT-scan. Int J Pediatr. 2019;7:9207-9213.

8. Kashio A, Sakamoto T, Karino S, Kakigi A, Iwasaki S, Yamasoba T. Predicting round window niche visibility via the facial recess using high-resolution computed tomography. Otol Neurotol. 2015;36:e18-e23.

9. Leong AC, Jiang D, Agger A, Fitzgerald-O'Connor A. Evaluation of round window accessibility to cochlear implant insertion. Eur Arch Oto-Rhino-Laryngol 2013;270:1237-1242

10. Park E, Amoodi H, Kuthubutheen J, Chen JM, Nedzelski JM, Lin VY. Predictors of round window accessibility for adult cochlear implantation based on pre-operative CT scan: a prospective observational study. J. Otolaryngol. Head Neck Surg. 2015;44:20.

11. Fouad YA, Elaassar AS, El-Anwar MW, Sabir E, Abdelhamid A, Ghonimy M. Role of multislice CT imaging in predicting the visibility of the round window in pediatric cochlear implantation. Otol Neurotol. 2017;38:1097-1103.

12. Su WY, Marion MS, Hinojosa R, Matz GJ. Anatomical measurements of the cochlear aqueduct, round window membrane, round window niche, and facial recess. The Laryngoscope. 1982;92:483-486. 